\title{
Review
}

\section{The fission yeast spindle orientation checkpoint: a model that generates tension?}

\author{
Yannick Gachet, Céline Reyes, Sherilyn Goldstone and Sylvie Tournier** \\ LBCMCP-CNRS UMR5088, Institut d'Exploration Fonctionelle des Génomes (IFR / 09), Université Paul Sabatier, I 8 route de Narbonne, \\ 31062 Toulouse, France
}

*Correspondence to: Sylvie Tournier, LBCMCP-CNRS UMR5088, Institut d'Exploration Fonctionelle des Génomes (IFR I09), Université Paul Sabatier, 118 route de Narbonne, 31062

Toulouse, France.

E-mail: tournier@cict.fr

Received: 20 June 2006 Accepted: 26 July 2006

\begin{abstract}
In all eukaryotes, the alignment of the mitotic spindle with the axis of cell polarity is essential for accurate chromosome segregation as well as for the establishment of cell fate, and thus morphogenesis, during development. Studies in invertebrates, higher eukaryotes and yeast suggest that astral microtubules interact with the cell cortex to position the spindle. These microtubules are thought to impose pushing or pulling forces on the spindle poles to affect the rotation or movement of the spindle. In the fission yeast model, where cell division is symmetrical, spindle rotation is dependent on the interaction of astral microtubules with the cortical actin cytoskeleton. In these cells, a bub1-dependent mitotic checkpoint, the spindle orientation checkpoint (SOC), is activated when the spindles fail to align with the cell polarity axis. In this paper we review the mechanism that orientates the spindle during mitosis in fission yeast, and discuss the consequences of misorientation on metaphase progression. Copyright (C) 2006 John Wiley \& Sons, Ltd.
\end{abstract}

Keywords: fission yeast; astral microtubule; spindle orientation; actin; mitosis

\section{Contents}

Introduction

Spindle positioning in fission yeast

Pre-anaphase astral microtubules: 'seeing is believing'

SOC vs. SAC: what is the difference?

Concluding remarks

References

\section{Introduction}

In metazoan cells, specification of the division plane and correct chromosome segregation requires the accurate control of spindle orientation during mitosis. Additionally, cell type diversity can be generated by a strategy involving 'regulated' orientation of the mitotic spindle, which dictates an asymmetric cell [46]. Model organisms have proved to be powerful tools for studying the mechanisms that control spindle orientation [29].
In particular, studies in the budding yeast Saccharomyces cerevisiae have contributed greatly to our present understanding of the general principles underlying the regulation of spindle positioning in asymmetrically dividing cells [24]. In this model, which unlike higher eukaryotic cells undergoes a closed mitosis, correct orientation of the spindle along the polarity axis, prior to the onset of anaphase, is essential for accurate chromosome segregation between mother and daughter cells. In $S$. cerevisiae, a precise temporal programme of astral microtubule-cortex interactions specifies spindle positioning, while the molecules that control bud site selection determine the axis of cell division. A peculiarity of $S$. cerevisiae is that spindle formation and positioning is initiated before the $\mathrm{G}_{1}-\mathrm{S}$ transition in the mother cell, therefore the nucleus and the spindle must migrate to the bud neck before alignment of the spindle to the axis of cell polarity (Figure 1, left panel). In contrast, in the fission yeast Schizosaccharomyces pombe, and in animal cells, spindle formation occurs only 


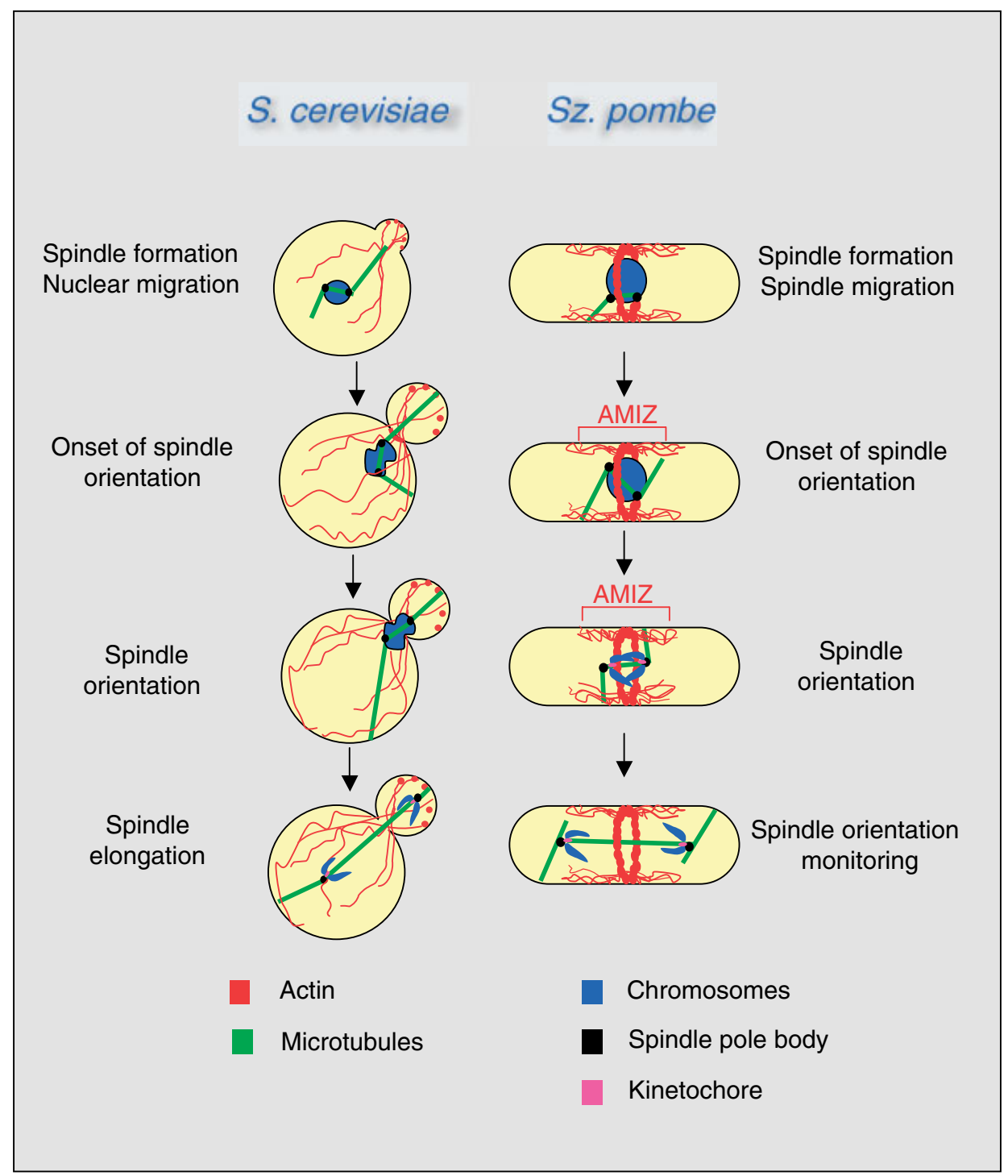

Figure I. Model illustrating the different phases in spindle orientation in budding and fission yeast. In the asymmetrically dividing budding yeast, nuclear migration towards the bud neck is the first step in spindle orientation and is rapidly followed by the pulling of the spindle through the bud neck. The onset of anaphase and spindle elongation occurs after these two steps. During the entire spindle orientation process, a precise programme of astral microtubule-cortex interactions is required. In the symmetrically dividing fission yeast, spindle positioning occurs in two phases. In the first of these phases, spindle migration, the spindle forms in close proximity to the cortex and aligns with the transverse axis of the cell. During the second phase, spindle orientation, which begins in metaphase and continues until early anaphase, the spindle corrects its angle with respect to the polarity axis of the cell. During spindle elongation, the alignment of the spindle with the axis of cell division is constantly monitored until the end of mitosis. Astral microtubules (green lines) that polymerize from the extranuclear face of the SPBs (black circles) interact initially with a zone that surrounds the nucleus, the AMIZ. Spindle migration, rotation and spindle orientation monitoring is driven by contact of astral microtubules with the cortex

in mitosis and does not require nuclear migration [36] (Figure 1, right panel). Furthermore, cell division is symmetric in $S z$. pombe, as is the case for most mammalian cell types. Thus, Sz. pombe provides an attractive eukaryotic system in which to study the mechanisms governing spindle orientation. It was initially thought that in $S z$. pombe spindle orientation could occur by sliding of the spindle poles along the cortex of the cell during spindle elongation. Indeed, spindle rotation can 
occur in the absence of astral microtubules as a consequence of pushing of the growing spindle against the cell wall $[16,41,63]$. This phenomenon, which we call orientation by force, only occurs after anaphase and is often seen in mutants deficient for spindle orientation [17]. Surprisingly however, we and others have shown that Sz. pombe uses a precise temporal programme of astral microtubule-cortex interactions to correct spindle misorientation $[16,17,41,63]$. A bub1dependent mitotic checkpoint that delays anaphase onset when spindle positioning is compromised has also been identified [65]. Since our original description of the spindle orientation checkpoint (SOC) in 2001, several advances have been made describing different aspects of spindle behaviour in response to perturbation of cell polarity, which we outline and discuss in this review.

\section{Spindle positioning in fission yeast}

\section{A role for early and late astral microtubules}

In $S z$. pombe the nucleus is centred in the middle of the cell in interphase by a microtubule-dependent mechanism (for review, see also Sawin and Tran in this issue). The cell then undergoes a symmetrical division that gives rise to two identical daughter cells after mitosis [20]. The fission yeast spindle is formed and positioned in $\mathrm{M}$ phase, and is composed of 12-16 microtubules that emanate from the two spindle pole bodies (SPBs), which are embedded on the opposite sides of a persistent nuclear envelope. These microtubules overlap in the central zone of the spindle, and an additional 10-12 microtubules originate from each SPB and terminate at the three pairs of sister kinetochores $[11,12,58]$. Visualization of live $S z$. pombe cells expressing GFP-tubulin has revealed that mitosis consists of three phases [39,59]. Phase 1 comprises prophase, during which a short $(\sim 2.0 \mu \mathrm{m})$ spindle is formed. In phase 2 the spindle maintains this length [76] and the centromeres make frequent, rapid movements between the poles, eventually congressing to form the metaphase plate [65]. At the end of phase 2, the sister chromatids separate (anaphase A) and move back to the SPBs. Phase 3 consists entirely of anaphase $\mathrm{B}$, during which the spindle elongates along the longitudinal axis of the cell to an eventual length of $\sim 14 \mu \mathrm{m}$.
The fission yeast SPB resides in the cytoplasm through most of interphase, where it duplicates. When the cell enters mitosis, the nuclear envelope invaginates beneath the SPB and forms a fenestra into which the duplicated SPB settles. Microtubules then polymerize from the inner plate of the SPB to build the spindle. As anaphase proceeds, the nuclear fenestrae close and the SPBs are extruded back into the cytoplasm [12]. Studies from fixed preparations have revealed that the cytoplasmic face of the two SPBs is associated with astral microtubules, which can exist in two configurations, termed convergent and parallel [21]. In either case, one end of each astral microtubule bundle is initially orientated towards the cortex at the cell mid-zone. Time-lapse analyses of mitotic cells expressing $\alpha$-tubulin-GFP has confirmed that astral microtubule interactions with the cell cortex and the actin ring are required for correct spindle orientation in fission yeast $[17,41,63]$.

In fission yeast, as in budding yeast, spindle positioning can be divided into three different phases (Figures 1 and 2). During prophase, the spindle forms in close proximity to the cortex and aligns along the polarity axis of the cell (spindle migration). In metaphase, astral microtubules initiate spindle rotation to align the spindle along the longitudinal axis of the cell until the angle between the spindle and the polarity axis is approximately $30^{\circ}$ [17]. This second phase of spindle positioning is rapidly followed by chromosome segregation and complete rotation of the spindle. Finally, during anaphase $\mathrm{B}$, astral microtubules emanating from both SPBs are required to correct the spindle angle until the end of mitosis (spindle orientation monitoring). Mechanical perturbations induced by laser ablation of post-anaphase astral microtubules have confirmed that astral microtubules are required to correct spindle orientation [63]. The sequence of these events is shown in Figure 2, where contact between the astral microtubules and the cortex can be visualized throughout mitosis, from the early phase of spindle migration through to anaphase B spindle orientation monitoring.

Several lines of evidence have suggested that functional astral microtubules are required for spindle orientation in fission yeast. First, all three phases of spindle alignment are perturbed in a cdc11-123 mutant, in which the nucleation of astral microtubules at the SPB is defective [28]. Second, mutations in the Slk19-related 


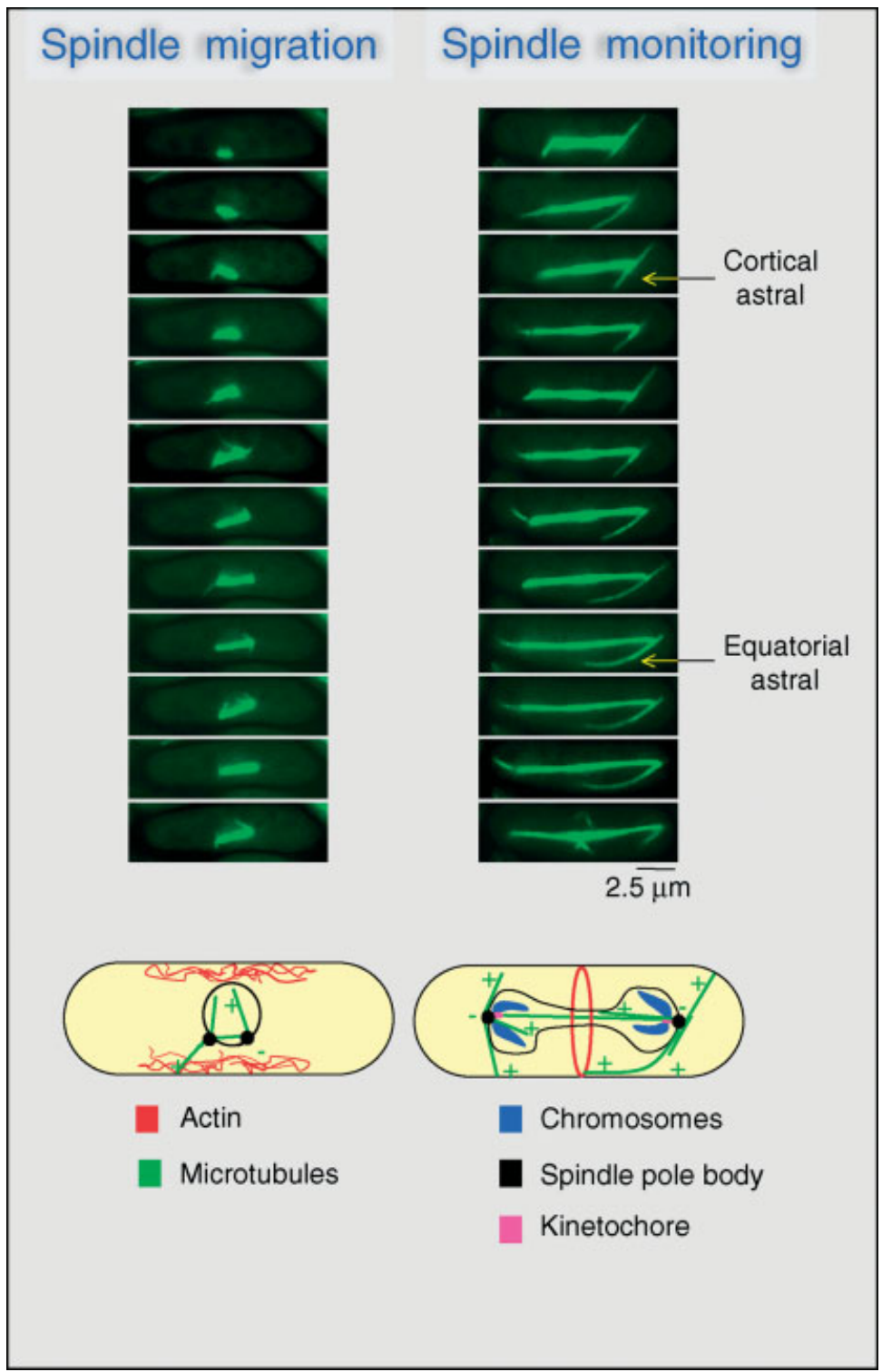

Figure 2. Astral microtubules are required for pre-anaphase spindle migration and post-anaphase spindle orientation monitoring. Live cell analysis of gfp-atb2 cells was performed using imaging chambers (CoverWell PCl-2.5, Grace Bio-labs) filled with $1 \mathrm{ml} \mathrm{l} \%$ agarose in minimal medium and sealed with $22 \mathrm{~mm}^{2}$ glass coverslips. Exponentially growing cells were applied to the imaging chamber, and the cells were allowed to equilibrate for $\mathrm{I} h$ at $24^{\circ} \mathrm{C}$ before beginning the experiments, which were carried out at the same temperature. Time-lapse images of a single-focal plane image were taken at $30 \mathrm{~s}$ intervals, with exposure times of $300 \mathrm{~ms}$ and the HBO lamp reduced to $30 \%$ to avoid photobleaching of astral MTs, as well as phototoxicity. Images were visualized with a Princeton CCD CooISNAP HQ camera fitted to a Leica DMI 6000 upright microscope with a $100 \times(1.33 \mathrm{NA})$ objective and were recorded using Metamorph. Astral MTs contact the cortex early in mitosis to position the spindle in the longitudinal axis of the cell (spindle migration). After anaphase onset, cortical astral microtubules are required to monitor spindle orientation until the end of mitosis

protein Alp7 affect mitotic spindle formation, astral microtubule function and spindle orientation [41,50,51]. Moreover, mutation of the non-essential protein mto1, which plays an important role in $\gamma$-tubulin complex-mediated microtubule nucleation, results in the failure to nucleate cytoplasmic astral microtubules and affects spindle orientation, although the assembly of the intranuclear mitotic 
spindle seems to be normal $[49,52,69,81]$. Finally, disruption of the plus-end microtubule-associated protein mal3 affects astral microtubule stability and spindle orientation (our unpublished data). The identification of the cortical cues required for astral microtubule capture at the cell cortex is now essential to unravel the subtleties of the mechanisms underlying spindle orientation in fission yeast.

\section{Cortical cues and spindle orientation}

The position of the cell division septum is dictated by the aniline homologue (an actin-binding protein) Mid1, which resides in the nucleus in interphase and relocates to the medial cell cortex in early mitosis $[3,9,42,56]$. As cells enter mitosis, actin disappears from the tips of the cells and relocalizes around the early mitotic nucleus to form the cytokinetic actomyosin ring (CAR), whose constriction directs the assembly of the cytokinetic septum [35]. More than 50 genes contribute to cytokinesis in $S z$. pombe. Several genes controlling actin dynamics have been shown to be essential for formation of the CAR. This is the case for Cdc15, a PST$\mathrm{PIP} / \mathrm{PCH}$ protein that localizes to the contractile ring [13]. Other non-essential proteins, such as the type $\mathrm{V}$ myosins Myo51 and Myo52, relocate from the cell tips to the CAR in metaphase [37,75] and are also required for the maintenance of cell polarity.

The different components of the CAR represent attractive candidates for cortical cues required for spindle orientation. Indeed, the plus-ends of the astral microtubules initially associate with the cell cortex in a $3 \mu \mathrm{m}$ wide medial band surrounding the nucleus, which we have termed the 'astral microtubule interaction zone' (AMIZ). Contacts with the AMIZ are initiated as the spindle forms, and continue during phase 2 of mitosis [17] (Figure 2). Live analysis of $c d c 15-g f p$ gfp$a t b 2$ cells has revealed that astral microtubules are captured at the AMIZ and then move towards the CAR, which is situated at the centre of the AMIZ. These findings suggest an essential role for the actin cytoskeleton in the regulation of spindle orientation. Indeed, spindle orientation is prevented by disruption of the medial actin cytoskeleton, either by addition of latrunculin (an actin depolymerizing drug) or in the actin mutant, cps8-188 [26]. Furthermore, the two type $\mathrm{V}$ myosins Myo51 and Myo52 are necessary for spindle rotation, as has also been described in budding yeast $[6,78]$. In contrast, other type myosins, e.g. Myp2 [75], do not seem to participate in the regulation of spindle orientation (our own unpublished observations). As spindle orientation is also deficient in cells deleted for For3 [17], a formin that nucleates actin cable assembly at the cell tips [15], it is tempting to speculate that astral microtubules contact actin cables at the medial cell cortex via the type $\mathrm{V}$ myosins to direct gross spindle rotation in fission yeast.

Strikingly, under all conditions in which spindle orientation is defective (cortical component mutants, SPB mutants, latrunculin treatment), the conformation of the astral microtubules is also perturbed. In wild-type cells, astral microtubules usually grow towards the equatorial or the cortical region (Figures 2, 3A), maintaining a relatively fixed angle to the spindle. In spindle orientation mutants, astral microtubules have different geometrical relationships with the cell cortex (our unpublished observations; Figure 3A). As is the case in higher eukaryotes, different categories of nonspindle microtubules exist in fission yeast cells, and it is likely that their regulation during the cell cycle and the molecular requirements for their function may be different (for review, see [8]). Indeed, Zimmerman and colleagues have recently described the existence of intranuclear microtubules (INA) that are present mainly during metaphase in fission yeast cells [82]. In Caenorhabditis elegans embryos, it has recently been shown that astral microtubules play two distinct roles in initiating cleavage furrow formation. In early anaphase, astral microtubules are required for contractile ring assembly; in late anaphase, astral microtubules show different cortical behaviour and seem to suppress cortical contraction at the poles [38]. Whether equatorial or cortical INAs and ENAs (extranuclear astral microtubules) have specific functions in fission yeast is at present unknown.

\section{Pre-anaphase astral microtubules: 'seeing is believing'}

Studying the mechanisms that control fission yeast spindle orientation requires the observation of pre-anaphase and post-anaphase extranuclear astral microtubules (ENAs). While stable postanaphase astral microtubules are easily visualized by either immunofluorescence or live microscopy 
A

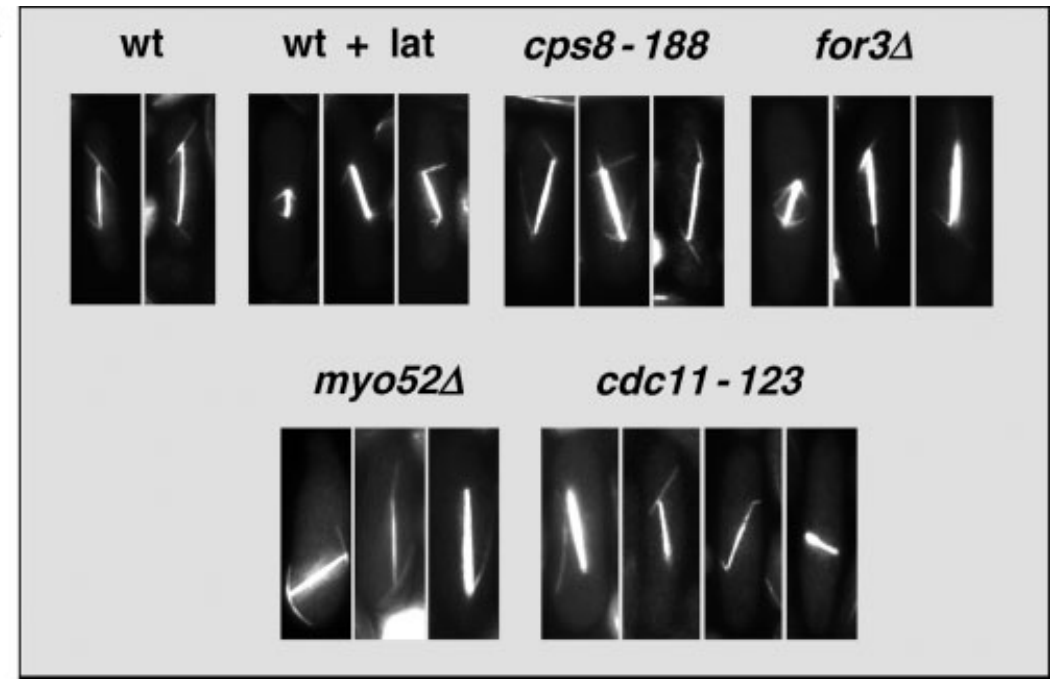

B

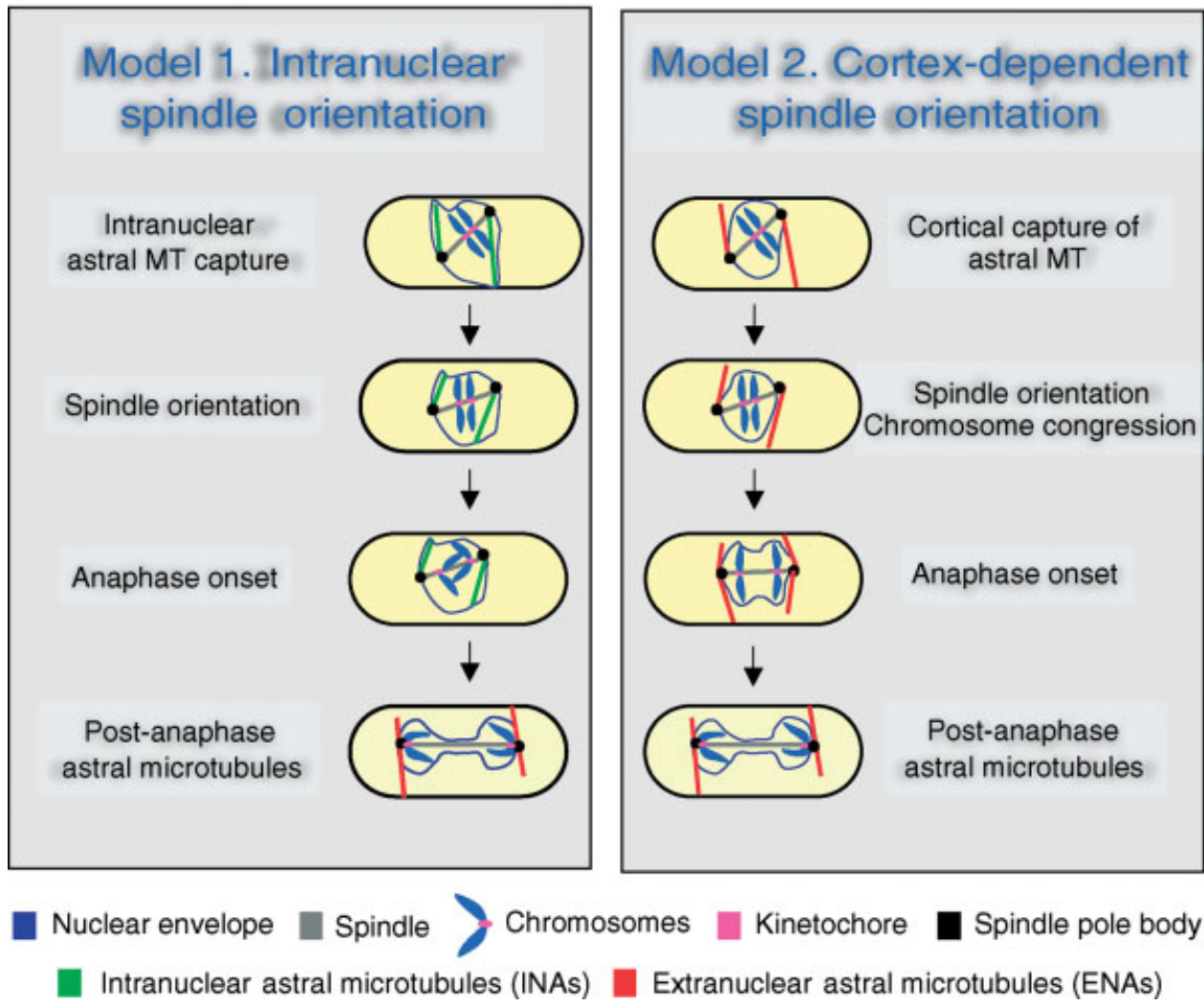

Figure 3. The different categories of non-spindle microtubules and their roles in spindle orientation. (A) Live cell analysis of gfp-atb2 cells was performed in different genetic backgrounds at $25^{\circ} \mathrm{C}$ : wild-type cells; wild-type cells treated with $10 \mu \mathrm{M}$ Lat B for $30 \mathrm{~min}$; the actin mutant cps8-188; the deletion of the formin For3; the deletion of the type $\mathrm{V}$ myosin Myo52; the mutant in the SPB outer plaque component $c d c /$ I-I23. Note that in wild-type cells, astral microtubules usually grow towards the equatorial or the cortical region, maintaining a relatively fixed angle to the spindle, while in the spindle orientation mutants the astral microtubules have different geometrical relationships with the cell cortex. (B) Illustration of the two possible models for pre-anaphase spindle orientation: an intranuclear or a cortex-dependent spindle orientation mechanism 
under most growth conditions, pre-anaphase astral microtubules are more dynamic and more sensitive to photobleaching and, as a result, have proved to be more elusive (our own observations). Recently, Zimmerman and colleagues have suggested that preanaphase astral microtubules do not exist and that only intranuclear astral microtubules are present in metaphase fission yeast cells [82]. This hypothesis raises two interesting questions.

First, how is the spindle positioned in metaphase without extranuclear astral microtubules? Second, how does the disruption of the cytoplasmic actin cytoskeleton influence metaphase spindle orientation if only intranuclear microtubules are present at this stage? Two models have so far been put forward (Figure 3B). In the first model, Zimmerman and colleagues hypothesize that INAs and the nuclear envelope participate in spindle positioning in fission yeast. According to this model, INAs could push and orientate on a rigid nuclear envelope. Alternatively, they could push and orientate on the cortex from within the nuclear envelope. In this case, orientation of the spindle before anaphase would require a high degree of flexibility in the nuclear envelope, and the stabilization of intranuclear microtubules after their capture by the nuclear envelope (Figure 3B, left panel). To validate this model, mutations affecting the presence and/or stability of INAs are necessary. In the second model, the INAs are not involved in spindle positioning during metaphase. Instead, the ENAs are required for both the early positioning and orientation of the spindle before the metaphase-anaphase transition (Figure 3B, right panel). Our own observations do not support the first model, as there seem to be no clear correlation between the presence of preanaphase INAs and spindle movement in wild-type cells (unpublished data). Additionally, the number and stability of the pre-anaphase INAs is increased in mutants with spindle defects, including misorientation or misattachment (our own unpublished data). Interestingly, using a similar methodology to that of Zimmermann et al. we were able to visualize ENAs during the early phase of spindle migration (phase I). The interaction of an astral microtubule with the cell cortex results in the movement of the metaphase spindle towards the centre of the cell, together with an invagination of the nuclear envelope (Figure 4). Although these observations suggest to us that pre-anaphase ENAs do indeed exist, visualization of the kinetochores, together with the spindle dynamics, is required to allow firm conclusions to be drawn regarding the presence or absence of preanaphase astral microtubules. However, as yet no laboratories have been able to provide this quality of analysis.

It is important to mention that the cortexdependent spindle orientation model does not exclude the presence of INAs, which we have observed and have been clearly described by others [48], but further work is necessary to define their role and the molecular requirements for their function. Finally, the necessity for astral microtubule contact with the cortex for spindle orientation is not unique to fission yeast, as this mechanism has already been established in many cell types: mouse embryos [34], Hela cells [62], NRK cells [40], $C$. elegans [30], Drosophila neuroblasts [18], the fungus Ustilago maydis [5] and budding yeast [77]. In these model systems, spindle orientation is determined before anaphase execution.

\section{SOC vs. SAC: what is the difference?}

During mitosis fidelity is the key, because chromosomes lost along the way generate aneuploidy, an event generally lethal during development. To avoid chromosome loss during mitosis, progression through the cell cycle is monitored by a series of checkpoints that ensure the temporal and spatial ordering of cell cycle events. One of the beststudied of these is the checkpoint that monitors the assembly of the mitotic spindle $[10,79,80]$. Components of this checkpoint, which is often referred to as the spindle assembly checkpoint (SAC), were first identified in $S$. cerevisiae and include the Mad1, Mad2, Mad3, Bub1, Bub3 and Mps1 proteins $[32,72]$. Structural and functional homologues of these proteins have been identified in all other eukaryotes so far examined, including $S z$. pombe $[7,23,25]$. In response to microtubule disrupting agents these proteins translocate to unattached kinetochores and inhibit the anaphase-promoting complex (APC) [44], an E3 ubiquitin ligase which is responsible for the destruction not only of cyclin B but also Securin, an inhibitor of Separase. Separase cleaves $\operatorname{Scc} 1 / \operatorname{Rad} 21$, a component of the cohesin complex which holds sister chromatids together [64,66,67] (Figure 5).

In an analogous manner, perturbation of the actin cytoskeleton delays entry into anaphase in fission 

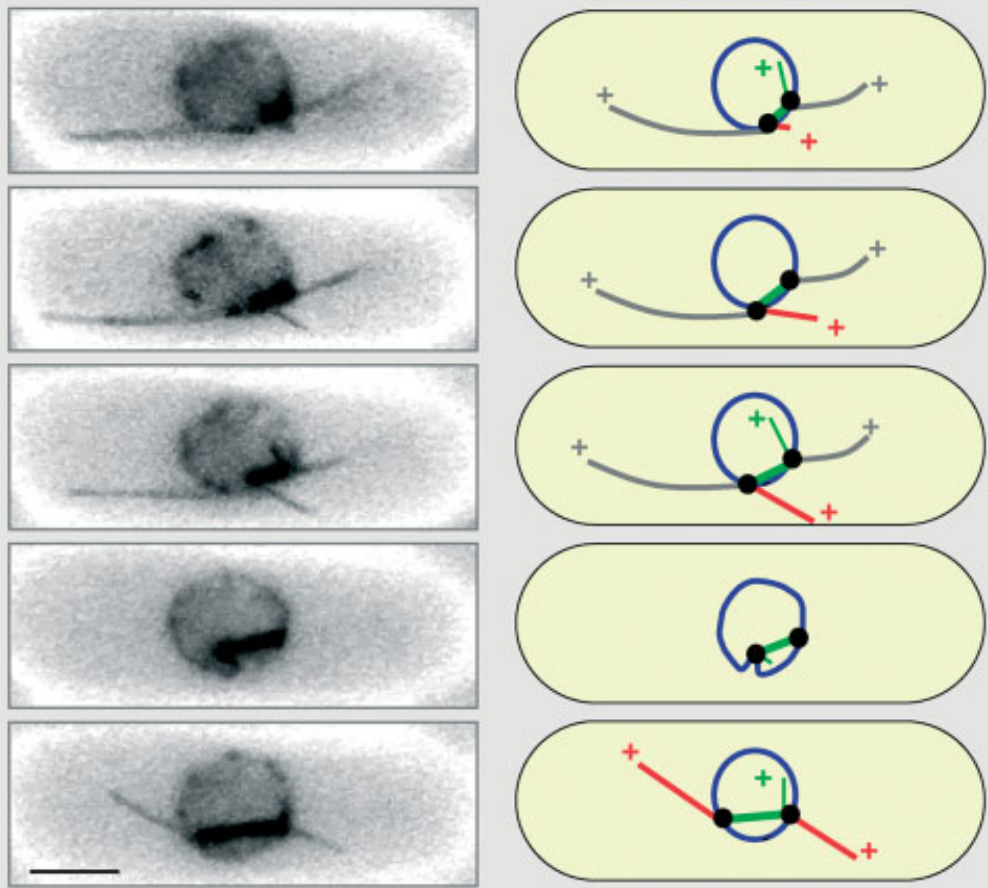

$2.5 \mu \mathrm{m}$

Nuclear envelope Cytoplasmic MTs

Intranuclear astral microtubules (INAs)

Extranuclear astral microtubules (ENAs)

Figure 4. Pre-anaphase INAs and ENAs in fission yeast. Live cell analysis of nup 107-gfp atb2-gfp cells was performed as described in Figure 2. Time-lapse images of a single-focal plane image were taken at $30 \mathrm{~s}$ intervals, with exposure times of $300 \mathrm{~ms}$ and the HBO lamp reduced to $30 \%$ to avoid photobleaching of astral MTs. Images were visualized with a Princeton CCD CooISNAP HQ camera fitted to a Leica DMI 6000 upright microscope with a $100 \times(1.33 \mathrm{NA})$ objective and were recorded using Metamorph. At the onset of mitosis, cytoplasmic microtubule bundles that had persisted from interphase were still present. ENAs appear during the early phase of spindle migration (phase I). The interaction of a pre-anaphase astral microtubule with the cell cortex results in the movement of the metaphase spindle towards the centre of the cell, together with an invagination of the nuclear envelope. At a later stage we also noticed the presence of ENAs on a $2.5 \mu \mathrm{M}$ spindle, but visualization of the kinetochores together with the spindle dynamics will be necessary to conclude that these are pre-anaphase astral microtubules. It will also be necessary to reproduce this observation using alternative nuclear envelope tagged components, as a defect in the functionality of nup 107-gfp has previously been described [4]

yeast cells. Cells treated with the actin depolymerizing agent latrunculin or cells carrying mutations in the actin gene enter mitosis and form a short, misoriented spindle with geometrically abnormal astral microtubules. Importantly, since latrunculin also delays the onset of sister chromatid separation, we have named this phenomenon the 'spindle orientation checkpoint' (SOC) [16]. In response to latrunculin, both the separation of sister chromatids and the degradation of spindle-associated 


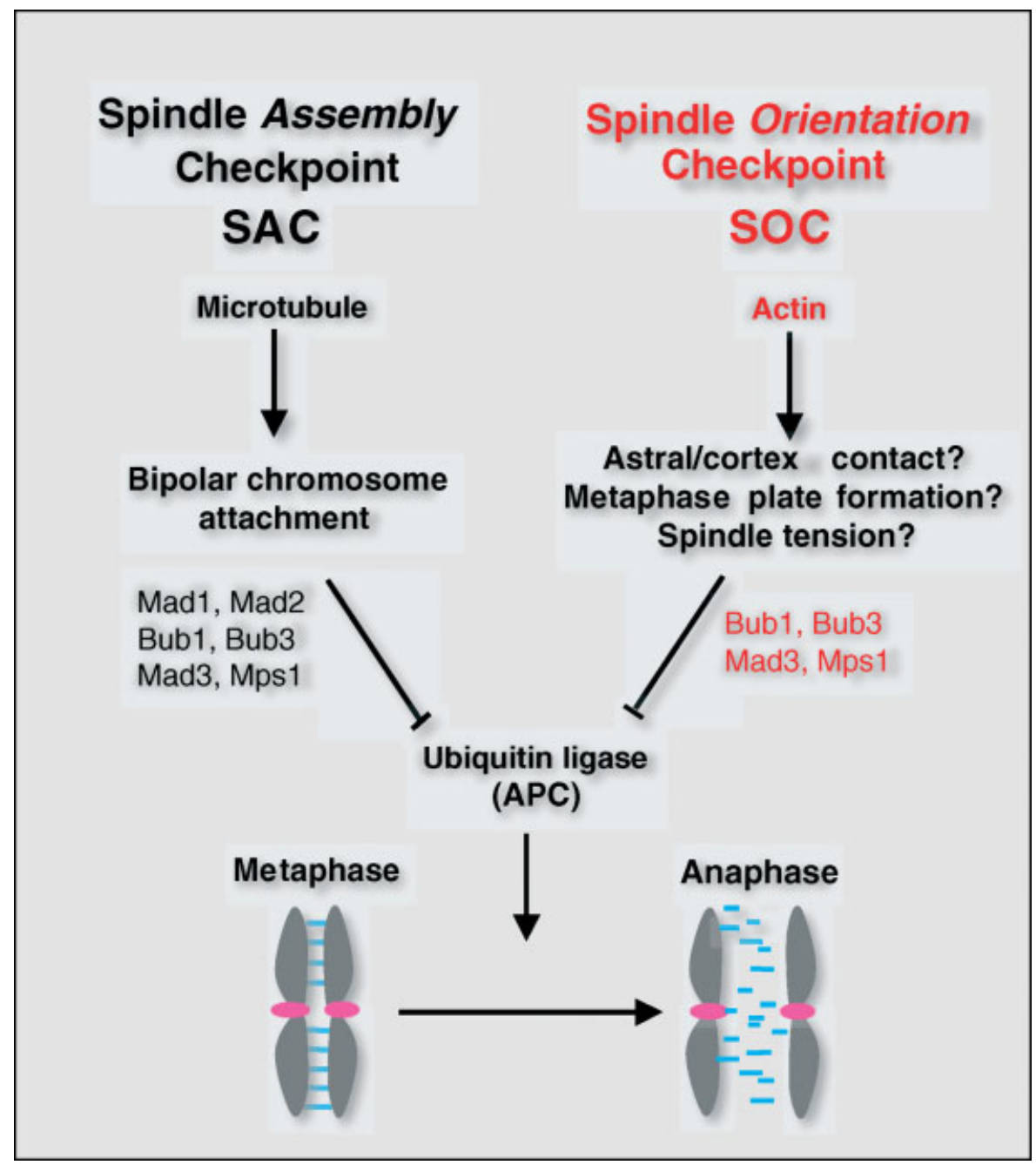

Figure 5. Mechanisms of activation of the SOC vs. the SAC. Microtubule depolymerizing drugs disrupt the bipolar attachment of kinetochores to the spindle and activate the SAC via the Mad I, Mad2, Mad3, Bub I, Bub3 and Mps I proteins. Actin-depolymerizing drugs disrupt astral microtubule interaction with the cell cortex and activate the spindle orientation checkpoint via the Bubl, Bub3 and Mad3 proteins. Whether the SOC senses an astral-cortex contact, the correct formation of a metaphase plate or correct tension across the kinetochores is still very much under debate

Cdc13 and Cut2 is delayed in cells lacking Mad1 or Mad2, but not in cells lacking Bub1, Mad3 or Bub3 [65]. Moreover, we find that addition of latrunculin A can impose an anaphase delay in cells lacking Mph1, although this is less pronounced than in wild-type cells. This may be because Mph1 is partially required for the recruitment of Bub1 to kinetochores [68].

Our results directly contradict the report by Rajapolan and colleagues who suggest that in fission yeast the metaphase spindle position dictates the timing of anaphase via Bub1 and Mph1 but not other SAC proteins [45]. This is a surprising result, since both Mad3 and Bub3 are necessary for Mps1 (Mph1) to impose a metaphase arrest in budding yeast in response to spindle damage [22]. Additionally, Bub3 is required for both the recruitment of Bub1 to the kinetochores and the checkpoint function of Bub1 in several species, including fission yeast $[14,19,61,68,70]$. One possible explanation of these results is that the metaphase delay observed in the pcp1D400-900 mutant (a kendrinlike SPB component) is due to a mitotic defect other than spindle misorientation, since spindle 
alignment is apparently not affected in this mutant [45]. Alternatively, since the timing of chromosome separation in this study was judged only by staining of nuclei and spindles, the timing of sister chromatid separation may have been masked by an additional delay in anaphase A.

In accordance with our observations, Bub1 but not Mad2 localizes to the kinetochores upon perturbation of the actin cytoskeleton. Importantly, anaphase onset and the disappearance of Bub1 from the kinetochores is also delayed in the actin mutant cps8-188, in mutants deleted for the two type V myosins Myo51 and Myo52 (present at the CAR in metaphase), in the formin deletion mutant for3 $\Delta$ (also present at the CAR in metaphase), and in the $c d c 11-123$ SPB mutant [17]. The cdc11 gene encodes for a component of the spindle pole body outer plaque, and is required for exit from mitosis (centriolin in human cells; NUD1 in budding yeast). The anaphase delay observed in these mutants is dependent on Bub1 but not Mad2.

At present, we believe that it is essential to isolate checkpoint components that are specific to the SOC vs. the SAC. To this end, we have screened a range of mutants for Lat $\mathrm{B}$ sensitivity and found that cells lacking Atf1, [54,74] a homologue of the human Atf2 transcription factor, are significantly more sensitive to latrunculin than wild-type cells. Atf1 is phosphorylated and activated by the Sty1 MAP kinase (also known as SPC1), a homologue of the mammalian p38 MAP kinase [73]. Interestingly, cells lacking Sty1 are also defective for the latrunculin-mediated mitotic delay. Furthermore, Sty1 is phosphorylated, activated and localized to the nucleus by addition of low doses of latrunculin, but not TBZ or benomyl, which are microtubule inhibitors that activate the SAC. Latrunculin treatment results in the expression of Atf1-dependent genes such as pyp2. Importantly, we found that, when synchronized, $\Delta$ atf1 cells completely failed to arrest nuclear division in the presence of latrunculin and that they lost viability as they passed through mitosis. These data suggest that the stressactivated MAP kinase pathway has an integral role in the SOC that prevents sister chromatid separation in response to perturbation of the actin cytoskeleton [16].

This hypothesis has been challenged by the work of Oliferenko and Balasubramanian [41], who have shown that deletion of Atf1 is also able to relieve the mitotic delay imposed by the SAC in a
Mia1/Alp7 mutant. Therefore, the function of the MAP kinase pathway may be required both for the SAC and the SOC to maintain sister chromatid cohesion. If this is the case, the significance of Sty 1 activation by drugs that depolymerize actin, but not microtubules, remains obscure. The answer may lie in the recent findings of Tatebe et al [60]. who suggest that the Tea4-Tea1 complex, together with the stress-signalling MAPK cascade, contribute to cellpolarity maintenance under conditions of stress. Alternatively, the recently established link between the MAP kinase pathway and the recruitment of polo kinase to the SPBs may explain some of these observations [43]. Certainly, establishing the role of the MAP kinase pathway in the control of cell polarity, MT dynamics and mitosis will provide new links between the extracellular environment and the cytoskeleton.

Clearly, further experiments are needed to clarify these issues and we can only conclude at present that the spindle assembly and the spindle orientation checkpoints both inhibit the APC by overlapping but non-identical mechanisms (Figure 5).

So what do the Bub1, Bub3 and Mad3 checkpoint proteins sense compared to Mad2 and Mad1? Maybe the answer lies within how mitotic checkpoint signalling is switched off. In the 'attachment' model, anaphase is initiated when all kinetochores are bound to a bipolar spindle [47]. In the 'tension' model, anaphase is initiated only when balanced tension is exerted on the kinetochores [33,57]. It has been difficult to distinguish between these two models, since tension obviously requires bipolar attachment and, conversely, the application of tension can enhance the overall occupancy of kinetochores and thus the stability of microtubule attachment [27]. If it was originally thought that Mad2 was the key effector of the SAC, more recent evidence seems to contradict this view. It has been suggested that Mad2 and BubR1 (Mad3) independently inhibit the APC in response to detachment of kinetochore microtubules or loss of spindle tension, respectively [55]. Treatment of HeLa cells with a low dose of vinblastine causes a mitotic delay without disrupting bipolar spindle formation, and with BubR1 $(\operatorname{Mad} 3)$, but not Mad2, retained at the kinetochores [55]. In agreement with this, BubR1 is present at the kinetochores as chromosomes congress to the metaphase plate, while Mad2 is not [71]. Conversely, it has been suggested that 
Mad2 and BubR1 act in a linear pathway to monitor spindle tension [53].

Finally, recent studies in fission yeast have revealed that the fission yeast EB1 homologue Mal3 cooperates with the Bub1-dependent spindle checkpoint, but does not require Mad2 function to prevent monopolar attachment [1]. Interestingly, as is the case for the SOC, the checkpoint proteins Bub1, Bub3 and Mad3, but not Mad1 and Mad2, are required to impose a mitotic delay in the mal3 $\Delta$ mutant [2]. Thus, the nature of the molecular events detected by the spindle assembly checkpoint and how this information is transmitted to the APC remains controversial. Consequently, at this point we can only hypothesize as to the nature of the signal sensed by Bub1 when the actin cytoskeleton is perturbed.

We find that Bub1, but not Mad2, associates with the kinetochores when astral microtubule contact with the medial cell cortex is disturbed, suggesting that the position of the spindle is sensed through the kinetochores. These results suggest that spindle positioning is coordinated with the timing of anaphase onset via a checkpoint that monitors spindle tension at the kinetochores $[45,65]$. One attractive possibility is that the SOC monitors the capture of astral microtubules at the CAR. However, this hypothesis has been directly challenged by the work of Zimmermann and colleagues [82], who find that astral microtubules only form after anaphase onset and as a consequence cannot participate in the SOC. Additionally, SOC components do not associate directly with the medial cell cortex, and Bub1 binds to the kinetochores in cells that have activated the SOC. Hence, it is more likely that spindle alignment is sensed at the kinetochores.

In fission yeast, the centromeres congress to the spindle mid-zone immediately prior to sister chromatid separation, suggesting that chromosome congression triggers anaphase onset. In the presence of latrunculin, or in mutants in which astral microtubule contact with the cell cortex is disturbed (including $c d c 11-123$ ), pre-anaphase kinetochore dynamics appear to be normal, suggesting that the kinetochores are attached and under tension. However, chromosome congression (metaphase plate formation) does not take place before anaphase, with the consequent presence of lagging chromosomes that usually move towards, and regain, the other chromosomes [65]. It will be interesting to determine whether kinetochore dynamics are also affected in the mtol mutant, which fails to nucleate cytoplasmic astral microtubules and present an increased rate of chromosome loss [52]. Interestingly, in contrast to Venkatram et al. [69], who described the presence of a mitotic delay in this mutant, other studies report few [81] or no [52] defects in mitosis.

One possibility is that a subset of SAC proteins, including Bub1, Bub3 and Mad3, not only ensure that the chromosomes congress to the spindle mid-zone (metaphase plate) but also that the spindle is positioned perpendicular to the cytokinetic actomyosin ring before anaphase onset. But how would actin disruption affect chromosome congression and mitotic progression? One possibility could be that actin, the formins and the type $\mathrm{V}$ myosins play a more direct role in spindle morphogenesis and chromosome congression, as has been recently proposed in cells with an open mitosis [31]. In this situation, astral microtubules would play no role in this checkpoint. Another possibility is that spindle movements directed by astral microtubules may facilitate chromosome congression and thereby trigger anaphase onset. If this is the case, the SOC in fission yeast may be indistinguishable from the checkpoint that ensures chromosome congression during metaphase.

\section{Concluding remarks}

This review illustrates the complexity of the mechanisms controlling spindle orientation in a symmetrically dividing cell. The remarkable finding is that these cells use a similar mechanism to the asymmetrically dividing budding yeast to orientate the spindle. Astral microtubule function is the key to this process. While defects in spindle orientation in fission yeast are not detrimental to life, perhaps because their consequences are either repaired or lead to aneuploidy and cell death in subsequent cell cycles, we believe that they become essential in multicellular dividing organisms, where the maintenance of genomic stability is critical for the organism as a whole. This has already been shown in a developmental context. While a similar spindle orientation checkpoint has not been formally described in higher eukaryotes, micromanipulation experiments performed by O'Connell and Wang [40] on NRK cells have shown that misoriented 
spindles underwent directed rotation, and that misorientation caused a delay in anaphase onset.

In symmetrically and asymmetrically dividing yeasts, when a defect in spindle orientation occurs, a mitotic delay is imposed to prevent genomic instability. In budding yeast, a Bub2/Cdc16-dependent delay is applied to prevent cytokinesis, while in fission yeast a Bub1-dependent and Cdc16-independent metaphase delay is observed
(Figure 6). This discrepancy may account for the timing differences in spindle morphogenesis in these two yeasts. Further experiments are needed to firmly establish that spindle orientation defects in fission yeast do not affect the timing of cytokinesis onset.

To understand what the SOC really senses: the integrity of the CAR, spindle positioning, astral microtubule contact with the cell cortex, or the
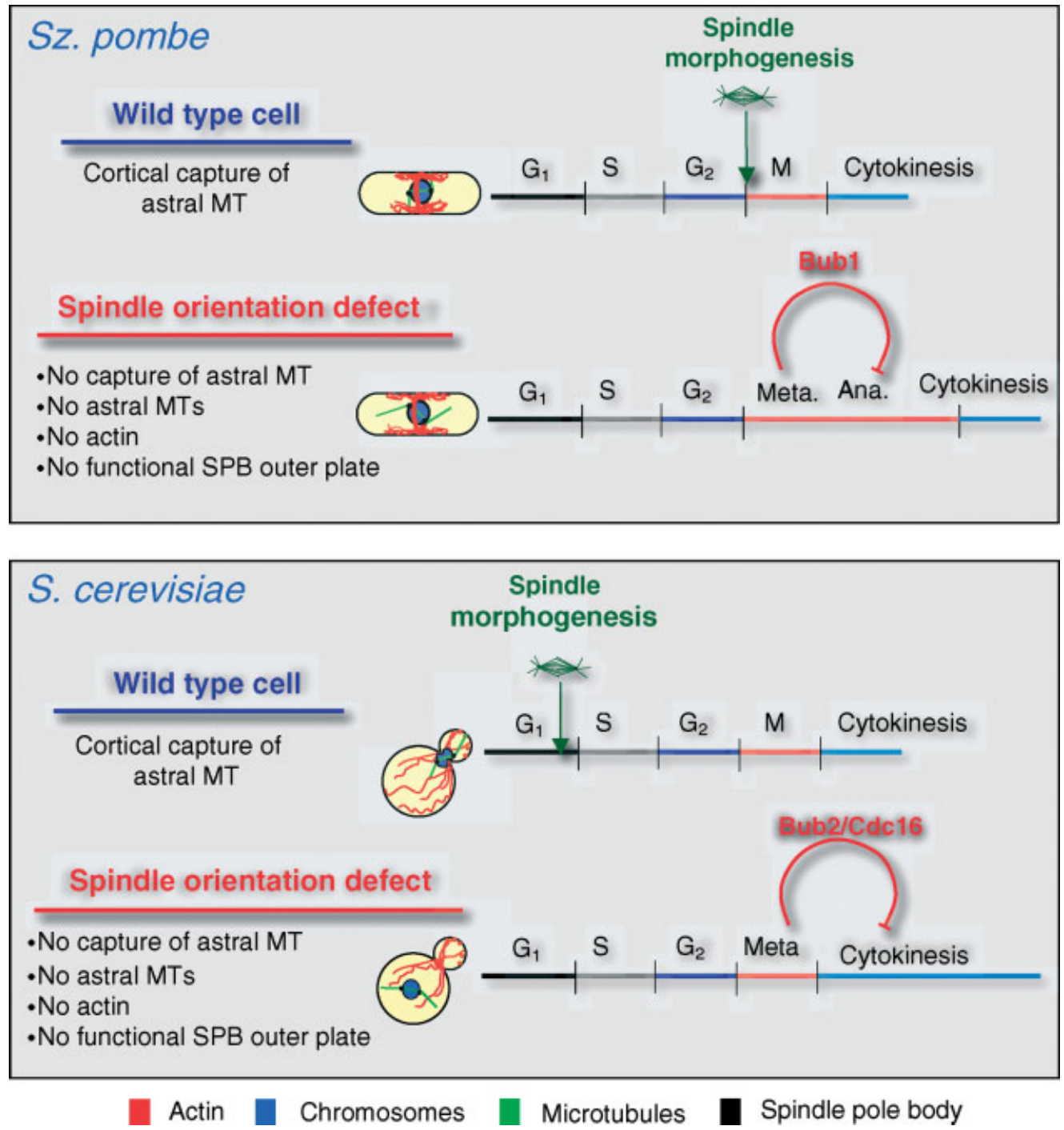

Figure 6. Similarity and differences in the control of spindle morphogenesis and positioning in budding and fission yeasts. In fission yeast, as in higher eukaryotes, the spindle forms at the onset of mitosis and both migration and orientation occur at the onset of mitosis. The position of the spindle is monitored throughout anaphase and a bubl-dependent mitotic delay is induced if misorientation is detected. In budding yeast, the spindle forms in $\mathrm{G}_{\mid}$and both nuclear migration to the bud neck and spindle orientation occur before the onset of anaphase. The position of the spindle is monitored by a Bub2-Cdcl6 pathway, which blocks the onset of cytokinesis if misorientation is detected 
correct establishment of a metaphase plate and tension across kinetochores, is a particularly difficult challenge because all of these events are intimately linked. Beyond this semantic argument, we believe that the most important finding is that the actin cytoskeleton may not only control spindle alignment but also contribute to the mechanisms underlying chromosome congression to the metaphase plate and, subsequently, the onset of anaphase.

\section{Acknowledgements}

We would like to thank Sir Paul Nurse, Professor Jeremy Hyams, Professor Mitsuhiro Yanagida and Professor Bernard Ducommun for helpful discussions and continuing encouragement; and Jurg Bahler, Valerie Wood and Joe Wixon for inviting us to write this review. Y.G. was supported by a post-doctoral fellowship from the Fondation pour la Recherche Médicale. Work in the laboratory is supported by the Association pour la Recherche sur le Cancer.

\section{References}

1. Asakawa K, Toda T. 2006. Cooperation of EB1-Mal3 and the Bub1 spindle checkpoint. Cell Cycle 5: 27-30.

2. Asakawa K, Toya M, Sato M, et al. 2005. Mal3, the fission yeast EB1 homologue, cooperates with Bub1 spindle checkpoint to prevent monopolar attachment. EMBO Rep 6 : 1194-1200.

3. Bahler J, Steever AB, Wheatley S, et al. 1998. Role of polo kinase and Midlp in determining the site of cell division in fission yeast. J Cell Biol 143: 1603-1616.

4. Bai SW, Rouquette J, Umeda M, et al. 2004. The fission yeast Nup107-120 complex functionally interacts with the small GTPase Ran/Spi1 and is required for mRNA export, nuclear pore distribution, and proper cell division. Mol Cell Biol 24: 6379-6392.

5. Banuett F, Herskowitz I. 2002. Bud morphogenesis and the actin and microtubule cytoskeletons during budding in the corn smut fungus, Ustilago maydis. Fung Genet Biol 37: 149-170.

6. Beach DL, Thibodeaux J, Maddox P, Yeh E, Bloom K. 2000. The role of the proteins Kar9 and Myo2 in orienting the mitotic spindle of budding yeast. Curr Biol 10: 1497-1506.

7. Bernard P, Hardwick K, Javerzat JP. 1998. Fission yeast bub1 is a mitotic centromere protein essential for the spindle checkpoint and the preservation of correct ploidy through mitosis. J Cell Biol 143: 1775-1787.

8. Burgess DR, Chang F. 2005. Site selection for the cleavage furrow at cytokinesis. Trends Cell Biol 15: 156-162.

9. Chang F, Woollard A, Nurse P. 1996. Isolation and characterization of fission yeast mutants defective in the assembly and placement of the contractile actin ring. J Cell Sci 109(1): $131-142$.

10. Cleveland DW, Mao Y, Sullivan KF. 2003. Centromeres and kinetochores: from epigenetics to mitotic checkpoint signaling. Cell 112: 407-421.
11. Ding R, McDonald KL, McIntosh JR. 1993. Three-dimensional reconstruction and analysis of mitotic spindles from the yeast, Schizosaccharomyces pombe. J Cell Biol 120: 141-151.

12. Ding R, West RR, Morphew DM, Oakley BR, McIntosh JR. 1997. The spindle pole body of Schizosaccharomyces pombe enters and leaves the nuclear envelope as the cell cycle proceeds. Mol Biol Cell 8: 1461-1479.

13. Fankhauser C, Reymond A, Cerutti L, et al. 1995. The $S$. pombe cdc15 gene is a key element in the reorganization of F-actin at mitosis. Cell 82: 435-444.

14. Farr KA, Hoyt MA. 1998. Bub1p kinase activates the Saccharomyces cerevisiae spindle assembly checkpoint. Mol Cell Biol 18: 2738-2747.

15. Feierbach B, Chang F. 2001. Roles of the fission yeast formin for $3 p$ in cell polarity, actin cable formation and symmetric cell division. Curr Biol 11: 1656-1665.

16. Gachet Y, Tournier S, Millar JB, Hyams JS. 2001. A MAP kinase-dependent actin checkpoint ensures proper spindle orientation in fission yeast. Nature 412: 352-355.

17. Gachet Y, Tournier S, Millar JB, Hyams JS. 2004. Mechanism controlling perpendicular alignment of the spindle to the axis of cell division in fission yeast. EMBO J 23: 1289-1300.

18. Giansanti MG, Gatti M, Bonaccorsi S. 2001. The role of centrosomes and astral microtubules during asymmetric division of Drosophila neuroblasts. Development 128: 1137-1145.

19. Gillett ES, Espelin CW, Sorger PK. 2004. Spindle checkpoint proteins and chromosome-microtubule attachment in budding yeast. J Cell Biol 164: 535-546.

20. Hagan I, Yanagida M. 1997. Evidence for cell cycle-specific, spindle pole body-mediated, nuclear positioning in the fission yeast Schizosaccharomyces pombe. J Cell Sci 110(16): 1851-1866.

21. Hagan IM, Hyams JS. 1996. Forces acting on the fission yeast anaphase spindle. Cell Motil Cytoskel 34: 69-75.

22. Hardwick KG, Weiss E, Luca FC, Winey M, Murray AW. 1996. Activation of the budding yeast spindle assembly checkpoint without mitotic spindle disruption. Science 273: 953-956.

23. He X, Patterson TE, Sazer S. 1997. The Schizosaccharomyces pombe spindle checkpoint protein $\operatorname{mad} 2 \mathrm{p}$ blocks anaphase and genetically interacts with the anaphase-promoting complex. Proc Natl Acad Sci USA 94: 7965-7970.

24. Huisman SM, Segal M. 2005. Cortical capture of microtubules and spindle polarity in budding yeast - where's the catch? J Cell Sci 118: 463-471.

25. Ikui AE, Furuya K, Yanagida M, Matsumoto T. 2002. Control of localization of a spindle checkpoint protein, $\mathrm{Mad} 2$, in fission yeast. J Cell Sci 115: 1603-1610.

26. Ishiguro J, Kobayashi W. 1996. An actin point-mutation neighboring the 'hydrophobic plug' causes defects in the maintenance of cell polarity and septum organization in the fission yeast Schizosaccharomyces pombe. FEBS Lett 392: 237-241.

27. King JM, Nicklas RB. 2000. Tension on chromosomes increases the number of kinetochore microtubules but only within limits. J Cell Sci 113(21): 3815-3823.

28. Krapp A, Schmidt S, Cano E, Simanis V. 2001. S. pombe cdc11p, together with sid4p, provides an anchor for septation initiation network proteins on the spindle pole body. Curr Biol 11: $1559-1568$. 
29. Kusch J, Liakopoulos D, Barral Y. 2003. Spindle asymmetry: a compass for the cell. Trends Cell Biol 13: 562-569.

30. Labbe JC, Maddox PS, Salmon ED, Goldstein B. 2003. PAR proteins regulate microtubule dynamics at the cell cortex in C. elegans. Curr Biol 13: 707-714.

31. Lenart P, Bacher CP, Daigle N, et al. 2005. A contractile nuclear actin network drives chromosome congression in oocytes. Nature 436: 812-818.

32. Li R, Murray AW. 1991. Feedback control of mitosis in budding yeast. Cell 66: 519-531.

33. Li X, Nicklas RB. 1995. Mitotic forces control a cell-cycle checkpoint. Nature 373: 630-632.

34. Louvet-Vallee S, Vinot S, Maro B. 2005. Mitotic spindles and cleavage planes are oriented randomly in the two-cell mouse embryo. Curr Biol 15: 464-469.

35. Marks J, Hagan IM, Hyams JS. 1986. Growth polarity and cytokinesis in fission yeast: the role of the cytoskeleton. J Cell Sci (suppl 5): 229-241.

36. McCully EK, Robinow CF. 1971. Mitosis in the fission yeast Schizosaccharomyces pombe: a comparative study with light and electron microscopy. J Cell Sci 9: 475-507.

37. Motegi F, Arai R, Mabuchi I. 2001. Identification of two type $\mathrm{V}$ myosins in fission yeast, one of which functions in polarized cell growth and moves rapidly in the cell. Mol Biol Cell 12: 1367-1380.

38. Motegi F, Velarde NV, Piano F, Sugimoto A. 2006. Two phases of astral microtubule activity during cytokinesis in $C$. elegans embryos. Dev Cell 10: 509-520.

39. Nabeshima K, Nakagawa T, Straight AF, et al. 1998. Dynamics of centromeres during metaphase-anaphase transition in fission yeast: Dis1 is implicated in force balance in metaphase bipolar spindle. Mol Biol Cell 9: 3211-3225.

40. O'Connell CB, Wang YL. 2000. Mammalian spindle orientation and position respond to changes in cell shape in a dynein-dependent fashion. Mol Biol Cell 11: 1765-1774.

41. Oliferenko S, Balasubramanian MK. 2002. Astral microtubules monitor metaphase spindle alignment in fission yeast. Nat Cell Biol 4: 816-820.

42. Paoletti A, Chang F. 2000. Analysis of midlp, a protein required for placement of the cell division site, reveals a link between the nucleus and the cell surface in fission yeast. Mol Biol Cell 11: 2757-2773.

43. Petersen J, Hagan IM. 2005. Polo kinase links the stress pathway to cell cycle control and tip growth in fission yeast. Nature 435: 507-512.

44. Pines J. 2006. Mitosis: a matter of getting rid of the right protein at the right time. Trends Cell Biol 16: 55-63.

45. Rajagopalan S, Bimbo A, Balasubramanian MK, Oliferenko S. 2004. A potential tension-sensing mechanism that ensures timely anaphase onset upon metaphase spindle orientation. Curr Biol 14: 69-74.

46. Rhyu MS, Knoblich JA. 1995. Spindle orientation and asymmetric cell fate. Cell 82: 523-526.

47. Rieder CL, Cole RW, Khodjakov A, Sluder G. 1995. The checkpoint delaying anaphase in response to chromosome monoorientation is mediated by an inhibitory signal produced by unattached kinetochores. J Cell Biol 130: 941-948.

48. Sagolla MJ, Uzawa S, Cande WZ. 2003. Individual microtubule dynamics contribute to the function of mitotic and cytoplasmic arrays in fission yeast. J Cell Sci 116: 4891-4903.
49. Samejima I, Lourenco PC, Snaith HA, Sawin KE. 2005. Fission yeast mto2p regulates microtubule nucleation by the centrosomin-related protein mtolp. Mol Biol Cell 16: 3040-3051.

50. Sato M, Koonrugsa N, Toda T, et al. 2003. Deletion of Mia1/Alp7 activates Mad2-dependent spindle assembly checkpoint in fission yeast. Nat Cell Biol 5: 764-766; author reply 766 .

51. Sato M, Vardy L, Angel Garcia M, Koonrugsa N, Toda T. 2004. Interdependency of fission yeast Alp14/TOG and coiled coil protein Alp7 in microtubule localization and bipolar spindle formation. Mol Biol Cell 15: 1609-1622.

52. Sawin KE, Lourenco PC, Snaith HA. 2004. Microtubule nucleation at non-spindle pole body microtubule-organizing centers requires fission yeast centrosomin-related protein mod20p. Curr Biol 14: 763-775.

53. Shannon KB, Canman JC, Salmon ED. 2002. Mad2 and BubR1 function in a single checkpoint pathway that responds to a loss of tension. Mol Biol Cell 13: 3706-3719.

54. Shiozaki K, Russell P. 1996. Conjugation, meiosis, and the osmotic stress response are regulated by Spc1 kinase through Atf1 transcription factor in fission yeast. Genes Dev 10: 2276-2288.

55. Skoufias DA, Andreassen PR, Lacroix FB, Wilson L, Margolis RL. 2001. Mammalian $\operatorname{mad} 2$ and bub1/bubR1 recognize distinct spindle-attachment and kinetochore-tension checkpoints. Proc Natl Acad Sci USA 98: 4492-4497.

56. Sohrmann M, Fankhauser C, Brodbeck C, Simanis V. 1996. The dmf1/mid 1 gene is essential for correct positioning of the division septum in fission yeast. Genes Dev 10: 2707-2719.

57. Stern BM, Murray AW. 2001. Lack of tension at kinetochores activates the spindle checkpoint in budding yeast. Curr Biol 11: $1462-1467$.

58. Tanaka K, Kanbe T. 1986. Mitosis in the fission yeast Schizosaccharomyces pombe as revealed by freeze-substitution electron microscopy. J Cell Sci 80: 253-268.

59. Tatebe H, Goshima G, Takeda K, et al. 2001. Fission yeast living mitosis visualized by GFP-tagged gene products. Micron 32: 67-74.

60. Tatebe H, Shimada K, Uzawa S, Morigasaki S, Shiozaki K. 2005. Wsh3/Tea4 is a novel cell-end factor essential for bipolar distribution of Teal and protects cell polarity under environmental stress in S. pombe. Curr Biol 15: 1006-1015.

61. Taylor SS, Ha E, McKeon F. 1998. The human homologue of Bub3 is required for kinetochore localization of Bub1 and a Mad3/Bub1-related protein kinase. J Cell Biol 142: 1-11.

62. Thery M, Pepin A, Dressaire E, Chen Y, Bornens M. 2006. Cell distribution of stress fibres in response to the geometry of the adhesive environment. Cell Motil Cytoskel 63: 341-355.

63. Tolic-Norrelykke IM, Sacconi L, Thon G, Pavone FS. 2004. Positioning and elongation of the fission yeast spindle by microtubule-based pushing. Curr Biol 14: 1181-1186.

64. Tomonaga T, Nagao K, Kawasaki Y, et al. 2000. Characterization of fission yeast cohesin: essential anaphase proteolysis of $\operatorname{Rad} 21$ phosphorylated in the S phase. Genes Dev 14: 2757-2770.

65. Tournier S, Gachet Y, Buck V, Hyams JS, Millar JB. 2004. Disruption of astral microtubule contact with the cell cortex activates a Bub1, Bub3, and Mad3-dependent checkpoint in fission yeast. Mol Biol Cell 15: 3345-3356. 
66. Uhlmann F, Lottspeich F, Nasmyth K. 1999. Sister-chromatid separation at anaphase onset is promoted by cleavage of the cohesin subunit Scc1. Nature 400: 37-42.

67. Uhlmann F, Wernic D, Poupart MA, Koonin EV, Nasmyth K. 2000. Cleavage of cohesin by the CD clan protease separin triggers anaphase in yeast. Cell 103: 375-386.

68. Vanoosthuyse V, Valsdottir R, Javerzat JP, Hardwick KG. 2004. Kinetochore targeting of fission yeast Mad and Bub proteins is essential for spindle checkpoint function but not for all chromosome segregation roles of Bub1p. Mol Cell Biol 24: $9786-9801$.

69. Venkatram S, Tasto JJ, Feoktistova A, et al. 2004. Identification and characterization of two novel proteins affecting fission yeast $\gamma$-tubulin complex function. Mol Biol Cell 15: 2287-2301.

70. Warren CD, Brady DM, Johnston RC, et al. 2002. Distinct chromosome segregation roles for spindle checkpoint proteins. Mol Biol Cell 13: 3029-3041.

71. Waters JC, Chen RH, Murray AW, Salmon ED. 1998. Localization of Mad2 to kinetochores depends on microtubule attachment, not tension. J Cell Biol 141: 1181-1191.

72. Weiss E, Winey M. 1996. The Saccharomyces cerevisiae spindle pole body duplication gene MPSI is part of a mitotic checkpoint. J Cell Biol 132: 111-123.

73. Wilkinson MG, Millar JB. 2000. Control of the eukaryotic cell cycle by MAP kinase signaling pathways. FASEB $J \mathbf{1 4}$ : 2147-2157.

74. Wilkinson MG, Samuels M, Takeda T, et al. 1996. The Atf1 transcription factor is a target for the Sty1 stress-activated
MAP kinase pathway in fission yeast. Genes Dev 10: 2289-2301.

75. Win TZ, Gachet Y, Mulvihill DP, May KM, Hyams JS. 2001. Two type $\mathrm{V}$ myosins with non-overlapping functions in the fission yeast Schizosaccharomyces pombe: Myo52 is concerned with growth polarity and cytokinesis, Myo51 is a component of the cytokinetic actin ring. J Cell Sci 114: 69-79.

76. Yamamoto A, West RR, McIntosh JR, Hiraoka Y. 1999. A cytoplasmic dynein heavy chain is required for oscillatory nuclear movement of meiotic prophase and efficient meiotic recombination in fission yeast. J Cell Biol 145: 1233-1249.

77. Yeh E, Yang C, Chin E, et al. 2000. Dynamic positioning of mitotic spindles in yeast: role of microtubule motors and cortical determinants. Mol Biol Cell 11: 3949-3961.

78. Yin H, Pruyne D, Huffaker TC, Bretscher A. 2000. Myosin V orientates the mitotic spindle in yeast. Nature 406: 1013-1016.

79. Yu H. 2002. Regulation of APC-Cdc20 by the spindle checkpoint. Curr Opin Cell Biol 14: 706-714.

80. Zhou J, Panda D, Landen JW, Wilson L, Joshi HC. 2002. Minor alteration of microtubule dynamics causes loss of tension across kinetochore pairs and activates the spindle checkpoint. J Biol Chem 277: 17200-17208.

81. Zimmerman S, Chang F. 2005. Effects of $\gamma$-tubulin complex proteins on microtubule nucleation and catastrophe in fission yeast. Mol Biol Cell 16: 2719-2733.

82. Zimmerman S, Daga RR, Chang F. 2004. Intra-nuclear microtubules and a mitotic spindle orientation checkpoint. Nat Cell Biol 6: 1245-1246. 